\title{
SEMEJANZAS ESTRUCTURALES ENTRE LA TRADICIÓN DE LA FILOSOFÍA MORAL Y LA TEORÍA SOCIAL DE DURKHEIM ${ }^{1}$
}

ANA MARTA GONZÁLEZ

Universidad de Navarra

RESUMEN: Las primeras teorías sociológicas son deudoras de las filosofías ilustradas de la historia, las cuales aparecieron para proporcionar un marco de sentido a la acción moral, una vez que la teoría moral renunció a los compromisos metafísicos de la filosofía moral premoderna. Al tiempo que defendió la autonomía de la sociología frente a la filosofía, Durkheim le prescribió a aquélla una tarea específica: realizar una ciencia moral que atendiendo a dos rasgos con los que los hechos morales se muestra a la conciencia - solidaridad y coerción - pueda dar cuenta del sentido moral de la división del trabajo, hasta entonces considerado un proceso casi natural. El propósito de este artículo es mostrar cómo el enfoque durkheimiano permite descubrir una semejanza estructural entre el análisis sociológico y la tradicional perspectiva filosófico-moral de las obligaciones mutuas.

PALABRAS CLAVE: coerción, división del trabajo, obligaciones, solidaridad, vínculo social.

\section{Structural similarities between the tradition of moral philosophy and Durkheim's social theory}

\begin{abstract}
The first sociological theories are indebted to Enlightenment philosophy of history, which first appeared to provide a framework of meaning for moral action once moral theory had renounced the metaphysical commitments of early modern moral philosophy. While defending the autonomy of sociology from philosophy, Durkheim prescribed a specific task for sociological thought, namely: develop a moral science which, by keeping together the two features with which moral facts appear before conscience - solidarity and coercion- could account for the moral sense of the division of labour, a phenomenon previously considered almost a natural process. The purpose of this article is to show how Durkheim's approach makes room for us to establish a structural similarity between sociological analysis and the perspective of mutual obligations characteristic of the moral philosophical tradition.
\end{abstract}

KEY WORDS: coercion, division of work, obligations, solidarity, social bond

1. LA DIFERENCIACIÓN DE FILOSOFÍA MORAL Y FILOSOFÍA DE LA HISTORIA EN LA GÉNESIS DE LA TEORÍA SOCIAL

Las primeras teorías sociales hunden sus raíces en la filosofía moderna de la historia, la cual, a su vez, es deudora de la evolución experimentada por la filosofía moral moderna, una vez que ésta abandona los compromisos metafísicos de la

1 Este trabajo iniciado en el marco del proyecto FFI2012-38737-C03-01 forma parte asimismo de los temas explorados en «Vínculos, emoción e identidad» (Ref. FFI2015-67388P), financiado por el Ministerio de Economía y Competitividad. 
filosofía moral precedente, y se ve en la tesitura de proporcionar un marco de sentido para la acción humana. El pensamiento de Kant es singularmente ilustrativo al respecto. La doble perspectiva kantiana sobre las acciones humanas -la perspectiva práctica del agente moral urgido por la ley, y la perspectiva especulativa del filósofo espectador de la historia-, representan, en realidad, dos momentos del proceso racional de un único sujeto que, a la hora de actuar, se ve en la necesidad de anticipar un horizonte de sentido.

Ciertamente, la anticipación de un horizonte de sentido para la acción, que reconocemos en Kant, se encontraba todavía más claramente en la teoría de la acción implícita en las aproximaciones aristotélica o tomista a la ética, en la medida en que iban precedidas por sendas indagaciones sobre el contenido de la eudaimonia o bienaventuranza. Sin embargo, tal indagación no constituía para ellos una investigación extrínseca a la reflexión moral propiamente dicha, pues no se limitaba a proporcionar un horizonte de sentido que hiciera posible orientar la acción del sujeto, sino que representaba un elemento necesario para justificar nuestro querer en general, y, consiguientemente, la misma realidad de la acción, de tal manera que, de no haber un Sumo Bien, fin último de todos nuestros actos, no habría acto voluntario de ningún tipo; no habría movimiento en general. La unidad de la razón aristotélica se aprecia singularmente en que, para Aristóteles, el momento especulativo de anticipación del fin se encuentra integrado en el momento práctico encaminado a realizar el sumo bien humano, que Aristóteles llamaba eudaimonia: lo que llamamos Sumo Bien es el nombre que damos al fin último, querido por sí mismo, y no querido a su vez por otra cosa, que da razón de nuestro querer en general (Cooper, 1977); por ello, ganar claridad en cuanto al contenido concreto del tal fin era el primer cometido de una investigación ética encaminada a realizar el bien del hombre.

En el planteamiento moral de Kant, por el contrario, el momento específicamente práctico, propio del agente ocupado en realizar el bien, aparece claramente diferenciado del momento hermenéutico a cuya luz aquella tarea moral adquiere último sentido. Por un lado, en efecto, tenemos la legislación práctico-moral y por otro la anticipación del horizonte hermenéutico, con el cual el sujeto trata la razón de la realidad social combinando las leyes de naturaleza y las leyes de la libertad. Ambos momentos, internamente diferenciados, definen los términos de dos empeños intelectuales bien distintos: por un lado, la filosofía moral, tal y como se ha cultivado en la época moderna, por otro las ciencias sociales.

En términos generales, la filosofía moral moderna está interesada en asegurar el orden social. Una vez que se ha desmoronado el orden medieval, se busca un fundamento filosófico de las normas que lo sustentan. Más allá de la diversidad de las fundamentaciones propuestas — paradigmáticamente la de Hume y la de Kant—al lector contemporáneo le sorprende la amplitud del consenso en cuestiones morales sustantivas, así como la coincidencia en alguna forma de universalismo moral (González, 2011b).

Ciertamente, en el caso de Hume, dicho universalismo no es absoluto, porque remite a la estructura psico-social de la naturaleza humana y es sostenido por una historia conjetural sobre los orígenes de la civilización (González, 2008; 2013), siendo por ello subsidiario de una filosofía de la historia que presenta el actual estado de cosas como el fruto maduro de una evolución histórica, plena de sentido. En el 
caso de Kant, en cambio, el universalismo moral, cuya realización histórica hemos de perseguir, remite a la razón, la cual prescribe una forma jurídica (González , 2010) y unos fines, por encima de toda diversidad empírica. Cabría decir que si en Hume la moral se explica la luz de la historia precedente, en Kant se propone como norma que ha de conducirnos a un futuro utópico.

En ambos casos, sin embargo, la conciencia moral del presente $-\mathrm{y}$, derivadamente, la experiencia de la diversidad cultural- se presta a ser explicada a la luz de la transición de un hipotético estado de naturaleza (que, en contraste con Hobbes, tanto Hume como Kant consideraban social) a un estado civilizado, dentro del cual se contempla una variedad de formas de vida, dependiendo de circunstancias geográficas, económicas, etc., más o menos consistentes con distintas formas políticas. Así, aunque la relación del estado civilizado y el progreso moral del hombre no siempre se interpreta en clave lineal (en el caso de Rousseau se interpreta más bien en clave de corrupción del hombre natural), en el siglo XIX, la noción de «civilización» llegará a marcar decisivamente la conciencia moral de Occidente (Elias), hasta constituirse en el núcleo inspirador de las primeras teorías sociales y antropológico-sociales, que, como es sabido, estaban marcadas por una aproximación evolutiva a la sociedad y la cultura. Una mirada retrospectiva a estas teorías muestra sus deudas con la filosofía de la historia precedente.

Por lo demás, la contextualización de la reflexión moral en aquel paradigma del abandono del estado de naturaleza pone de manifiesto que, en ausencia de los referentes metafísicos pre-modernos, el orden moral que la filosofía moderna trataba de fundamentar de iure sólo podía afirmarse de facto mediante su inserción en una filosofía de la historia, que justificara las aparentes variaciones de la norma en atención a una consideración diacrónica de la vida social: la moral racional sólo podía sostenerse, de hecho, bien merced a la fuerza de la costumbre y las historias conjeturales sobre el origen de las sociedades modernas (Berry, 1997), bien proyectándola hacia un futuro utópico. Desvinculada de ese marco histórico o utópico, la filosofía moral quedaba abocada a una dialéctica inevitable, entre modelos racionales-abstractos de fundamentación y concesiones más o menos razonables a la sensibilidad moral dominante.

Lo que Kant pudo justamente reprochar a Hume es que ni el sentimiento moral, ni la combinación de sentimiento y artificio alcanzan a dar razón de la universalidad y necesidad característica del deber moral. Por el contrario, resaltar la especificidad del hecho moral, del hecho del «deber», que, como una ley, se impone a la conciencia, era la principal virtualidad de la filosofía moral kantiana, y lo que ha llevado a considerarla paradigma de éticas deontológicas, capaz de enfrentarse a modelos teleológicos. No obstante lo anterior, Kant suscribe también una filosofía de la historia en la que, además de poner de manifiesto todas las ambigüedades morales que rodean al proceso civilizatorio, se subraya, de distintas maneras, la discrepancia creciente entre cultura y felicidad (González, 2004a; 2011a; 2014).

Precisamente, el modo en que Kant afronta la constelación moralidad-felicidadcultura, ofrece un notable punto de contraste con el optimismo del planteamiento utilitarista, para el cual lo prescrito por la norma moral corre en paralelo con lo requerido por la felicidad y la cultura. Al mismo tiempo, la aproximación kantiana representa también un contrapunto anticipado a la teoría social de Herbert Spencer, quien, dentro de su aproximación evolutiva a la realidad social, consideraba 
que el proceso de diferenciación social, propiciado por la división del trabajo, debería arrojar un saldo final positivo en términos de armonía social, con independencia de que el motor de la agencia individual fuera el interés (Lukes, 1984: 142). Si bien Kant también propone una armonía final de moralidad y felicidad, el modo en que lo hace - como postulado de la razón práctica, coherente con el deber $a$ priori de realizar el sumo bien- deja bien patente la distancia que existe no sólo entre moralidad y felicidad, sino la que existe también entre las leyes «naturales» que rigen el desarrollo histórico y son en buena parte responsables de la cultura, y las «leyes morales» que deben regir el comportamiento humano.

El pensamiento de los siglos XIX y xx tomará nota de esas distancias de distintas maneras: como malestar de la cultura (Freud), como tragedia de la cultura (Simmel), como alienación (Marx). Èmile Durkheim habla directamente de crisis moral, y la interpreta como anomia (Durkheim, 1967: 8), relacionándola con el mal de infinito que aqueja a los individuos del fin de siglo (Múgica, 2005c: 28), y que se manifiesta en la crisis del vínculo social (Mestrovic, 1991: 93). De estos modos tan diversos, pues, el pensamiento de finales del XIX y principios del xx levantaba acta de la crisis de la modernidad. En su peculiar formulación de la crisis, sin embargo, la aproximación desde la teoría social resultaba especialmente apremiante porque daba a entender que el malestar, la alienación, o la anomia, procedían del mismo proceso al que se atribuía el progreso social del último siglo, a saber, la división del trabajo.

Por esta razón, para Durkheim resultará prioritario mostrar que la división del trabajo, a la que se debe la diferenciación funcional, no era sólo una «ley natural» del desarrollo de las sociedades, análoga por lo demás a la que se registra en los organismos biológicos, sino que tenía también una significación moral, en el sentido de que, en su mismo desarrollo, favorece alguna forma de vínculo moral (Durkheim, 1967: 8-9). Desde esta perspectiva, tal y como ha observado Bryan S. Turner, la mayor preocupación de Durkheim consistió en encontrar un sistema de regulación o coerción moral que fuera relevante en las condiciones modernas; preocupación que le condujo a considerar la evolución de los sistemas de códigos profesionales y valores cívicos que podían contribuir a regular la economía, de modo similar a como los gremios lo habían hecho en las condiciones de la sociedad medieval (Turner, 1996: xiv).

Ahora bien: al proponerse la tarea de mostrar que la división del trabajo tiene también una significación moral, Durkheim imprimió un giro a la teoría social: la sacó del marco natural-evolutivo en el que la había situado Spencer —en último término deudor de una filosofía de la historia más o menos grandiosa- y la remitió al terreno de los conceptos morales y, con ello, de alguna manera, al terreno de la moral vivida.

\section{El RETORNO DE LA MORAL A LA TEORÍA SOCIAL}

Ciertamente este retorno de la teoría social al terreno de la moral vivida todavía no es completamente visible en Durkheim, al menos en el sentido de que su modo de abordar el hecho social deja en un segundo plano la perspectiva del agente 
individual, en beneficio de las regularidades a las que el observador de los «hechos sociales» trata de dar una significación moral. Como sabemos, para recuperar la perspectiva del agente tenemos que mirar a Weber (González, 2016a), si bien, dicha perspectiva sólo llegará a constituir el núcleo mismo de la consideración de la vida social con Schütz y el interaccionismo simbólico.

En este trabajo, sin embargo, no nos interesa tanto resaltar la moralidad incorporada en la perspectiva del agente —en último término por su orientación al bien como fuente de sentido- cuanto la regulación que emerge de la misma estructura de las relaciones humanas, definiendo las condiciones de la misma vida social. Bien y normas constituyen dos principios de la vida moral, siempre en perpetua tensión, que pueden ser acentuados diversamente. En el caso de Durkheim, el acento, al menos en un primer momento, recae en el aspecto normativo (Hall, 1993).

Según él mismo hace notar en el prefacio a la segunda edición de la División del Trabajo Social (1893), a Durkheim le preocupaba que la falta de regulación moral de la vida económica resultara en una desmoralización general de la sociedad:

«Si, en las ocupaciones que ocupan casi todo nuestro tiempo, no seguimos más regla que la de nuestro interés bien entendido, ¿cómo tomaríamos gusto por el desinterés, por el olvido de sí, por el sacrificio? Así, la ausencia de toda disciplina económica no puede dejar de extender sus efectos más allá del mundo económico mismo y de traer como consecuencia una disminución de la moralidad pública» (Durkheim 1967: 9).

Ya la sola formulación de este problema permite apreciar una curiosa inversión de la relación que filosofía moral y teoría social habían mantenido hasta entonces: mientras que en la edad moderna la filosofía de la historia, precedente de la teoría social, había proporcionado el marco de sentido para el desarrollo de la filosofía moral, el planteamiento de Durkheim da a entender que las razones morales —más que la filosofía moral propiamente dicha- proporcionan un referente inexcusable para la propia teoría social, la cual no puede trabajar simplemente sobre la hipótesis de que los hombres son maximizadores de la utilidad, prescindiendo de su condición de agentes morales: ya se conciba la moral como un contrapunto a la racionalidad instrumental en el seno de las interacciones humanas, o como núcleo coercitivo y/o solidario del vínculo social, la teoría social no puede prescindir de ella en su explicación de la sociedad humana sin distorsionar peligrosamente su objeto.

No obstante haber favorecido también el ideal de una ciencia social a semejanza de las ciencias naturales (Kant, 1994a; González, 2009), el propio Kant habría adelantado en parte la consideración de la moral como un elemento central en la explicación de la sociedad humana, pues, a su juicio, lo que da razón del desarrollo histórico no es otra cosa que la realización de la vocación moral del hombre (González, 2014). Sin embargo, en la medida en que Kant había sustraído el hecho moral a la racionalidad científico-positiva, había cerrado el camino a una reflexión sociológica centrada en la moral propiamente dicha. Indirectamente, esto había dejado expedito el camino para que el utilitarismo se convirtiera en el punto de referencia ético para las nacientes ciencias sociales (Mill, 2002: 81-2). 
Ahora bien, mientras que el utilitarismo propugnaba la subordinación de la moralidad a la felicidad, y un crecimiento en paralelo de felicidad y civilización, a Durkheim «no le era evidente que la transformación de un tipo estructural societario generara un sistema de vida ética en que los seres humanos fueran más felices; más generosos y altruistas; menos egoístas y calculadores. No eran tan evidentes las repercusiones inmediatas que en el plano de la vida ética podían tener, por ejemplo, el comercio, el aumento de la riqueza, la sofisticación de los gustos, los modales y las costumbres, etc.» (Múgica, 2004a: 11).

La civilización resultante de la división del trabajo no podía pensarse como un efecto intencionado de la acción individual, sino más bien como un efecto estructural sobrevenido al desarrollo de la vida social; un efecto que, desde el punto de vista moral, resultaba a simple vista ambivalente — como también lo era para Kant-. Sin embargo, para Durkheim sostener la ambivalencia moral de la cultura no podía significar la ausencia de toda relación entre civilización y moral, ni comportar un permanente desplazamiento del ideal moral hacia el futuro, como si no fuera posible reconocerle realidad histórica alguna. Incluso Kant, para quien resultaba imposible afirmar con certeza que hubiera habido alguna vez un acto moralmente puro, reconocía un signo del ideal moral en el entusiasmo que generan algunos hechos históricos — como la reacción de simpatía que la revolución francesa suscitó entre espectadores contemporáneos- (Kant, 1994 b: 88; González, 2014); y dejaba de considerar el derecho, al menos en parte, como un símbolo de la moral.

Lejos de pensar que la división del trabajo abocaba irremediablemente a la anomia, Durkheim se propuso demostrar que avanzaba una forma específica de cohesión moral (Durkheim, 1967: 10, 12). Este proyecto se encuadraba dentro de un planteamiento más general, que —bajo la influencia de los moralistas alemanes del XIX (Durkheim, 1975; Mestrovic, 1991: 96; Lukes, 1984: 92) - comprendía hacer de la moral una ciencia positiva, Science des Moeurs, pero de signo diverso al utilitarismo; una ciencia que acogiera la especificidad de los hechos morales, ya destacada por Kant, pero que, a diferencia de Kant — que situaba lo moral en un cielo trascendental, despegado de la historia- permitiera una aproximación positiva. En esta línea escribe: «Este libro es ante todo un esfuerzo por tratar los hechos de la vida moral según el método de las ciencias positivas (...) no queremos extraer la moral de la ciencia, sino hacer la ciencia de la moral, lo que es muy diferente» (Durkheim, 1967: 33)

Ciertamente, en la pretensión de realizar una ciencia de la moral resuenan las distintas posturas que a finales del XIX servirán al propósito de demarcar la sociología de la filosofía, sobre el fondo de una herencia y preocupaciones comunes (Pinto, 2009: 25; Lukes, 1984: 66). Además, en el caso particular de Durkheim, resue-na, especialmente, la influencia de Renouvier (Lukes, 1984: 54). En todo caso, sobre este trasfondo, tal y como ha argumentado Robert T. Hall, Durkheim podría ser caracterizado como un teórico moral, o un sociólogo de la moral, cuya teoría social general podría considerarse desde la perspectiva de la moral (Hall, 1993: 12), un estudioso de la sociedad guiado por el doble propósito de desarrollar una sociología moral y proponer una teoría ética (Hall, 1993: 15). El propio Hall considera que la primera gran obra de Durkheim, la División del Trabajo, fue concebida en estos términos, y también su segunda gran obra, El Suicidio, respondía al mismo plan (Hall, 1993: 16). Cuando murió en 1917 había empezado a editar sus lecciones 
de la Sorbona en una obra de tres volúmenes, titulada «La Moral», que incluía sus lecciones sobre moral cívica y profesional, su obra sobre la familia y muchos de los análisis incluidos en sus lecciones sobre educación moral (Hall, 1994: 21).

De cualquier forma, el punto de partida de esa "science des moeurs» suponía identificar algún rasgo empírico significativo de lo moral, «encontrar el sesgo por donde (los hechos) resultan científicos, es decir, descubrir en ellos algún elemento objetivo que implique una determinación exacta, y, si es posible, la medida» (Durkheim 1967: 35-6). Concretamente, Durkheim buscará los rasgos definitorios de los fenómenos morales en dos direcciones: solidaridad y coerción (Lukes, 1984: 111-14). Hechos sociales — por tanto coercitivos - con proyección solidaria. Para Durkheim, en efecto, "la moralidad es esa fuerza colectiva que tiene la capacidad de preservar el orden societario sin que ello implique de suyo una amenaza a la libertad. La fuerza de la moral es su carácter solidario y, sobre todo, su condición obligatoria, que no contraría, sin embargo, el libre consentimiento» (Múgica, 2004 a: 113; Lukes, 1984: 412-3).

Aunque la alusión a la solidaridad nos habla de la influencia de Schopenhauer — tan subrayada por Mestrovic (Mestrovic, 1991: 110; 1992)_, tal cosa no anula a mi juicio la influencia estructural del planteamiento kantiano. Ésta se advierte - como ya se ha indicado- no tanto en el deseo de hacer de la moral una ciencia algo que para Kant resultaría imposible- cuanto en el tomar solidaridad y coerción como indicios de lo moral y, en consecuencia, tomar el derecho como símbolo de la moral, todo lo cual venía insinuado en la reflexión kantiana sobre el entusiasmo.

\section{LA DIMENSIÓN MORAL DEL VÍNCULO SOCIAL}

Implícita en el planteamiento de Durkheim se encuentra la idea de que el orden social no es puramente voluntarista — fruto de pactos políticos- ni revolucionario; así como tampoco fruto de la simple prolongación del interés individual, o la coordinación estratégica de intereses individuales (Múgica, 2004b: 5-11):

«La moral —escribe- es para nosotros un sistema de hechos realizados, ligado al sistema total del mundo. Ahora bien, un hecho no se cambia fácilmente, ni siquiera si es deseable. Por otra parte, como es solidario con otros hechos, no puede ser modificado sin que éstos sean alcanzados, y a menudo es muy difícil calcular por adelantado el resultado final de esta serie de repercusiones» (Durkheim, 1967: 35-6).

Para Durkheim, el orden social es esencialmente moral y no simplemente el producto natural de la racionalidad estratégica practicada por agentes auto-interesados. En este contexto resulta significativo que, en su defensa de las corporaciones profesionales como fuentes de solidaridad y de moral, siguiendo los estudios de Waltzing, descarte como razón original de las corporaciones romanas la búsqueda del propio interés y, en cambio, situé en primer plano sus vinculaciones religiosas:

«Sin duda, la asociación les daba más fuerzas para salvaguardar en la necesidad sus intereses comunes. Pero no era ésta más que una de las consecuencias útiles que producía la institución; lo que no era la razón de ser, la función principal. Ante todo, la corporación era un colegio religioso. Cada una de ellas tenía 
su dios particular cuyo culto, cuando tenía los medios para ello, se celebraba en un templo especial. Así como cada familia tenía su Lar familiaris, cada ciudad su Genius publicus, cada colegio tenía su dios tutelar, Genius collegii. Naturalmente, este culto profesional no se daba sin fiestas, que se celebraban en común por sacrificios y banquetes. Todo tipo de circunstancias servía, por otra parte, de ocasión para alegres reuniones... Como corolario de este carácter religioso, el colegio de artesanos era, simultáneamente, un colegio funerario» (Durkheim, 1967: 14-5).

Aun diferenciándose de las corporaciones romanas, los gremios medievales —observa Durkheim, siguiendo a Levasseur — también manifestaban un mismo espíritu de solidaridad.

«El lugar tan considerable que la religión ocupaba en su vida, tanto en Roma como en el Medievo — concluye Durkheim — hace particularmente evidente la naturaleza verdadera de sus funciones; pues toda comunidad religiosa constituía entonces un medio moral, así como toda disciplina moral tendría forzosamente a tomar una forma religiosa... Ahora bien, esta unión con algo que supera al individuo, esta subordinación de los intereses particulares al interés general es la fuente misma de toda actividad moral. Si este sentimiento se precisa y se determina, si, aplicándose a las circunstancias más ordinarias y a las más importantes de la vida, se expresa en fórmulas definidas, tendremos un cuerpo de reglas morales en vías de constitución» (Durkheim, 1967: 17).

Ahora bien: defender esta interpretación moral-solidarista de las corporaciones profesionales significaba enfrentarse con la extendida interpretación utilitaria que, a la hora de dar cuenta del comportamiento económico, privilegiaba como motivo natural el interés. Frente al individualismo subyacente a estas interpretaciones, Durkheim sostenía que:

«la vida común es atractiva, al mismo tiempo que coercitiva. Sin duda, la obligación es necesaria para llevar al hombre a superarse a sí mismo, a agregar a su naturaleza física otra naturaleza: pero a medida que aprende a gustar los encantos de esta existencia nueva, contrae la necesidad de ella, y no hay orden de actividad donde no la busca apasionadamente. He aquí por qué los individuos que encuentran poseer intereses comunes se asocian, no sólo para defender sus intereses, sino para asociarse, para no sentirse perdidos en medio de adversarios, para tener el placer de comunicarse, de fundirse con otros, es decir, en definitiva, para llevar juntos una misma vida moral» (Durkheim 1967: 18).

Estas palabras nos recuerdan otras muy semejantes de Hume - no en vano el escocés trató de argumentar, frente a Hobbes, que, al lado de pasiones egoístas, hay en el hombre pasiones sociales-, pero con ellas Durkheim perfila una visión del orden social completamente distinta a la propuesta por el utilitarismo, presunto heredero del pensador escocés ${ }^{2}$. Con ello, Durkheim se enfrentaba a la línea de pensamiento social dominante en su época — representada por Spencer-, que consideraba el progreso civilizatorio en continuidad con el desarrollo natural.

En efecto: como es sabido, Spencer trataba de dar cuenta del orden social aplicando principios extraídos de la biología: diferenciación funcional, evolución y

2 En realidad la teoría moral escocesa no es pura y simplemente utilitarista; de hecho, no sólo la teoría económica neo-clásica, sino también la teoría económica institucionalista —Veblen, Galbraith—puede remitirse a la herencia escocesa. (González, 2013: 231) 
supervivencia del más apto. Desde estos principios aventuraba un futuro utópico, en el que los individuos ya no experimentarían la discrepancia entre la satisfacción de sus deseos individuales y el cumplimiento de su funcionalidad social. Con ello Spencer planteaba una teoría social en la que, al amparo de una visión organicista se daban cita el principio natural-evolutivo y una teoría de la acción individualista, en la que cada agente se mueve persiguiendo su interés, en un proceso cuyo resultado final estaría marcado por la integración social y la aparición de un nuevo tipo de personalidad, en perfecta armonía con las necesidades sociales (Spencer, 1966: 598-601).

El propio Durkheim, que, por influencia de los moralistas alemanes, Alfred Espinas y, en general, por el ambiente spenceriano que dominaba la reflexión sociológica de la época, comenzó próximo a posiciones organicistas —como todavía se advierte en la División del Trabajo $(1893)^{3}$ o en Las reglas del método sociológico (1895)—, sin embargo mantuvo desde el principio una postura crítica frente a ese planteamiento, por entender que falseaba la naturaleza moral del vínculo social, al suponer que el organismo social podría generar espontáneamente la integración social aun persiguiendo cada individuo su propio interés (Lukes, 1984: 82). En El Suicidio (1897), y, sobre todo, en Las Formas elementales de la vida religiosa (1912), el distanciamiento del organicismo es más claro, en la medida en que se da más entrada a la psicología en la explicación de los fenómenos sociales. Pero este giro no modificó su aproximación esencialmente moral a la vida social, que es una constante en toda su obra, y que impedía una visión puramente pragmática de la vida social, como la favorecida por algunas corrientes del pensamiento moral moderno.

En efecto: en contraste con una aproximación aristotélica de la sociedad, como realidad intrínsecamente ética, una aproximación típicamente moderna como la de Hume da pie fácilmente a una visión puramente pragmática de la vida social (González, 2016b: 40-41). La misma aproximación kantiana a las instituciones permite una explicación puramente naturalista y pragmática de su génesis (González. 2004b), al menos cuando se considera desde el punto de vista de filosofía de la historia. A la luz de estos precedentes modernos, no sorprende que la división del trabajo fuera considerada por Spencer como una «ley natural del desarrollo social», por mucho que, como advirtiera el propio Adam Smith, fuera una ley con importantes repercusiones morales, promotora, incluso, de cruciales transformaciones en la misma estructura psicológica de los hombres. Para Durkheim, en cambio, la división del trabajo no podía ser solo una ley natural de la evolución social sino que debía tener un significado moral, si es que la vida social había de tener justificación alguna.

\section{El SIGNIFICAdO MORAL DE LA DIVISIÓN DEL TRABAJO}

Precisamente ésta es la cuestión que recogió Durkheim, cuando, a finales del siglo XIX escribió su tesis sobre La división del trabajo: le interesaba explicar por

3 Así escribe: «(La división del trabajo) no es sólo una institución social que tiene su fuente en la inteligencia y en la voluntad de los hombres; es un fenómeno de biología general, cuyas condiciones es necesario buscar, parece, en las propiedades esenciales de la materia organizada». (Durkheim, 1967: 40-1) 
qué, en las sociedades modernas, el individuo se vuelve simultáneamente más autónomo y más dependiente de la sociedad; simultáneamente más personal y más solidario (Durkheim, 1967: 37).

Esta cuestión, sin embargo, formaba parte de una problemática más general, cuyo sentido último enunciábamos más arriba: si la división del trabajo es una simple ley «natural», o si tiene también un significado moral, de tal manera que la sociedad resultante de la división del trabajo, pese a todas las diferencias que podamos reconocer entre ella y las sociedades precedentes, no deje de exhibir rasgos morales —-solidarios-, ni deje de generar formas de vida compatibles con el carácter moral.

La ley de la división del trabajo — que Durkheim describía entonces como ley biológica - afecta a las sociedades humanas de manera inevitable, influyendo de manera decisiva en nuestra propia constitución moral. Con ello, sin embargo, no está todo decidido en términos morales. Tal y como él hace notar,

«El desarrollo del hombre se hará en dos sentidos muy diferentes, según que nos abandonemos a este movimiento o que nos resistamos a él. Pero entonces se plantea una cuestión urgente: ¿Cuál de las dos direcciones hay que tomar? ¿Nuestro deber es acaso volvernos un ser acabado y completo, un todo que se basta a sí mismo, o, por el contrario, no ser más que la parte de un todo, el órgano de un organismo?»(Durkheim, 1967: 41).

Con esas palabras Durkheim evocaba un ideal que en su época ya estaba en franca retirada: el ideal del hombre completo, «que, sabiendo interesarse por todo sin dedicarse exclusivamente a nada, capaz de gustar y comprender todo, que encontraba un medio de reunir y condensar en sí lo que había de más exquisito en la civilización», estaba siendo ampliamente superado por el curso de los acontecimientos, que más bien favorecen la aparición de especialistas disciplinados, frente a los cuales aquel otro tipo humano queda rebajado a la categoría de "aficionado", e incluso se nos antoja antisocial (Durkheim, 1967: 42).

Ahora bien, ¿en qué sentido se puede afirmar que la especialización derivada de la división del trabajo tiene justificación moral? Para responder a esta cuestión, Durkheim llama la atención sobre la solidaridad social basada en diferencias, y se pregunta hasta qué punto dicha solidaridad contribuye a la integración en sociedades complejas. Entiende que de ello depende advertir la cualidad moral de la división del trabajo: "si la función de la división del trabajo es tal, debe tener un carácter moral, pues las necesidades de orden, de armonía, de solidaridad social pasan generalmente por ser morales» (Durkheim, 1967: 60).

Según esto, examinar las variaciones históricas y sociales de la moral equivaldría a examinar cómo se ven afectadas las fuentes de la solidaridad y la coerción. Ahora bien: dado que por su propia naturaleza el vínculo solidario es algo interior que se escapa a la observación, para examinar aquellas variaciones históricas según criterios científicos no hay más remedio que atender a un hecho exterior en el que se refleje simbólicamente tal vínculo. Durkheim cree encontrarlo en el derecho (Durkheim, 1967: 61).

Durkheim observa cómo «todo derecho escrito tiene un doble objeto: prescribir ciertas obligaciones y definir las sanciones que están ligadas a ellas. En el derecho civil, y más generalmente en toda clase de derecho con sanciones restitutivas, el 
legislador aborda y resuelve separadamente los dos problemas. Determina, primero, la obligación con toda precisión posible y sólo entonces dice la manera cómo debe ser sancionada... El derecho penal, por el contrario, solamente dicta sanciones, pero nada dice de las obligaciones a las que aquéllas se refieren... Sólo puede haber para esto una razón: que todo el mundo conoce y acepta la norma» (Durkheim, 1967: 70-71).

En el caso del derecho penal, Durkheim advierte que «el crimen hiere los sentimientos que, para un mismo tipo social, se encuentran en todas las conciencias sanas» (Durkheim, 1967: 69). El protagonismo que adquieren los sentimientos morales no es el punto menos importante en el que su planteamiento recuerda al de los moralistas escoceses, lo cual no es extraño, puesto que pretende una aproximación empírica a la moral. De todos modos, Durkheim se ve obligado a matizar:

«No definimos al crimen cuando dijimos que consiste en una ofensa a los sentimientos colectivos, pues hay entre estos últimos algunos, que pueden ser ofendidos sin que haya crimen... Los sentimientos colectivos a los cuales el crimen corresponde deben, pues, diferenciarse de los otros por alguna propiedad distintiva: deben tener una cierta intensidad media. No sólo están grabados en todas las conciencias, sino que están fuertemente grabados. No son veleidades titubeantes y superficiales, sino emociones y tendencias que están fuertemente enraizadas en nosotros. Lo prueba la extrema lentitud con que evoluciona el derecho penal» (Durkheim, 1967:72-3).

Para Durkheim, la rigidez del derecho penal es significativa de la fuerza de los sentimientos colectivos que protege. Por el contrario, la rapidez con la que evolucionan otras reglas, muestran la menor energía del sentimiento que las sanciona. Con todo, la intensidad de los sentimientos no es suficiente criterio para que las conductas de los que los han ofendido entren en el catálogo del derecho penal; además — dice— tales sentimientos han de ser precisos, pues también son intensos los sentimientos que provoca la falta de piedad filial y no se sancionan como crimen (Durkheim, 1967: 73-4).

En todo caso, lo que el derecho penal refleja, para Durkheim, no es otra cosa que «el conjunto de creencias y de sentimientos comunes al término medio de los miembros de una sociedad»; para él, ese conjunto "forma un sistema determinado que tiene vida propia: podemos llamarlo conciencia colectiva o común», si bien, «ésta no tiene por substrato un órgano único: por definición es difusa en toda la extensión de la sociedad», pero "completamente distinta a las conciencias particulares, aunque sólo se realice en los individuos» (Durkheim, 1967:74).

Como es sabido, la naturaleza de la «conciencia colectiva», a la que Durkheim se refiere en las palabras anteriores, ha sido uno de los puntos más debatidos de su propuesta ${ }^{4}$, no tanto porque sustancialice la conciencia colectiva al margen de los individuos - acaba de señalar que aquélla no se realiza más que en los individuos-, sino porque no especifica el modo en que sobrepasa a los individuos, ni el modo en que éstos la hacen propia.

\footnotetext{
4 «No hay que decir que un acto ofende la conciencia común. No lo reprobamos porque es un crimen, sino que es un crimen porque lo reprobamos» (Durkheim, 1967: 75). Adviértase: a) la similitud con planteamientos morales voluntaristas; b) la inversión de la teleología — típicamente espinosista — implicada en la primacía de la tendencia sobre su objeto. (Durkheim, 1967: 76).
} 
Según Durkheim esa «conciencia colectiva» no debe identificarse con toda conciencia social, la cual, en sus propias palabras, «se extiende tan lejos como la vida psíquica de la sociedad», pues en las sociedades superiores, la conciencia colectiva "sólo es una parte muy restringida». De hecho, en las sociedades desarrolladas, todas las funciones especiales - gubernamentales, científicas, etc. - son de orden psíquico, "porque consisten en sistema de representaciones y de acciones: sin embargo, evidentemente, están fuera de la conciencia común». (Durkheim, 1967: 74-5).

Tal cosa se corresponde con el hecho de que, en las sociedades superiores, caracterizadas por una intensa división del trabajo, aumente el protagonismo del derecho restitutivo frente al derecho penal, lo cual es indicativo del tipo de solidaridad característica de tales sociedades, una solidaridad orgánica, basada no en similitudes sino en diferencias funcionales:

«En efecto, está en la naturaleza de las tareas especiales escapar a la acción de la conciencia colectiva; pues, para que una cosa sea objeto de sentimientos comunes, la primera condición es que sea común, es decir, que esté presente en todas las conciencias y que todas puedan representársela desde un solo e idéntico punto de vista. Sin duda, en cuanto las funciones conserven cierta generalidad todo el mundo puede tener algún sentimiento: pero cuanto más se especializan, más se circunscribe el número de aquellos que tienen conciencia común. Las normas que la determinan no pueden tener, por lo tanto, esa fuerza superior, esa autoridad tan trascendente que, cuando es ofendida, reclama expiación. En gran parte su autoridad proviene de la opinión, tal como ocurre con las normas penales, pero de una opinión localizada en regiones restringidas de la sociedad. Además, aun en los círculos especiales donde se aplican y donde en consecuencia están representadas en los espíritus, no corresponden a sentimientos muy vivos, ni aun, a menudo, a ninguna especie de estado emocional»(Durkheim, 1967: 111).

Con lo anterior, Durkheim ha mostrado la relación existente entre división del trabajo y la aparición de un tipo distinto de diferenciación social, expresiva de un diverso vínculo solidario y moral. También aquí, en efecto, se trata de un vínculo moral, puesto que también se encuentran presentes los dos rasgos de solidaridad y coerción de los que se sirve para identificar el fenómeno moral (Durkheim, 1967: 394). Llegados a este punto, sin embargo, nos interesa dar un paso atrás y ver en qué sentido esta clase de aproximación al fenómeno moral no resulta ajena al planteamiento clásico.

\section{LA PERSPECTIVA DE LAS OBLIGACIONES MUtUAS}

En efecto: el lenguaje y, sobre todo, el marco novedoso con el que Durkheim se refiere a lo moral no debe hacernos pasar por alto las semejanzas de lo que plantea con los temas tradicionales de la filosofía moral. Después de todo, la aproximación a la moral desde la perspectiva de la coerción y la solidaridad se encuentra implícita en la aproximación al orden moral en términos de deberes; una aproximación que contempla la vida social desde las perspectiva de las obligaciones mutuas - legales y morales - que estructuran o perfeccionan la vida social. En estos términos, en efecto, se presenta la tradicional división de los deberes en legales y morales, y la ulterior división de estos últimos en deberes sin cuyo cumplimiento no puede haber 
sociedad y aquellos otros que se refieren a la honestidad de las costumbres (Tomás de Aquino, 1997: q.80; González, 2011b). Por ello analizar las variaciones históricas de lo moral equivale a analizar las variaciones en la institucionalización social y la fuerza motivadora de los distintos deberes, en los que se recoge la perspectiva de las obligaciones mutuas, y se reflejan la estructura misma de la vida social y las exigencias del bien común.

La perspectiva de las obligaciones mutuas no sólo permite abordar la dimensión moral de la vida social, sino que también permite subrayar la dimensión social de la vida moral, en mayor medida que una ética de virtudes, que se presentara exclusivamente en términos de auto-perfección del sujeto.

Ciertamente, la definición aristotélica de la virtud como «el hábito por el cual el hombre se hace bueno y por el cual ejecuta bien su función propia» (Aristóteles, 1985: 25; EN, II, 6, 1106a) no tiene por qué conducir a una ética narcisista, principalmente porque el agente humano, para Aristóteles, es un agente naturalmente social. Sin embargo, incluso entonces, y precisamente por eso, una aproximación integral a la vida moral necesita ser complementada con la referencia a la ley, como institución que, desde fuera del agente, introduce orden en la vida social.

En efecto: el hecho de que el ser humano sea naturalmente un ser social quiere decir que no todo el orden social tiene su fuente en el hombre en cuanto ser individual; quiere decir que el bien del individuo va a depender, al menos en parte, de un orden que no tiene directamente su origen en el mismo individuo, y que - por eso mismo- - va a ser experimentado por él como cierta coerción. Hablar de coerción en este contexto no tiene nada que ver con el hecho de que el individuo en cuestión no pueda realizar el comportamiento requerido de buena gana, e incluso por iniciativa propia, atraído por su bondad o belleza. Tiene que ver, simplemente, con el hecho de que la definición de esa acción que él realiza tal vez de buena gana y por propia iniciativa tiene su origen en una fuente exterior a él mismo. Según Durkheim, esa fuente es la sociedad (Durkheim, 1967: 86). Por ello él trata de acceder a los fenómenos morales explorando distintas formas de coerción social.

A ello cabe objetar que no toda forma de coerción social es por eso significativa de un fenómeno moral. Y, de hecho, Durkheim dice que sólo las que tienen una virtualidad solidaria lo son. Aunque esta última acotación parecería dejar fuera de juego el capítulo de los deberes para con uno mismo, no hay que descartar de antemano que también estos deberes puedan ser reconstruidos como teniendo un origen social y un fin solidario. En este sentido, Durkheim escribe que «los deberes del individuo para consigo mismo son, en realidad, deberes para con la sociedad; corresponden a ciertos sentimientos colectivos que ya no está permitido ofender cuando el ofendido y el ofensor son una misma y sola persona, ni cuando son dos seres distintos». (Durkheim, 1967: 395). Según esto, en su planteamiento, la sociedad aparece como el medio moral por excelencia, incluso aunque el origen y el destinatario de sus exigencias sea el mismo individuo.

A su vez, afirmar que la sociedad sea el medio moral por excelencia no significa necesariamente constituirla en el último fin de la vida moral; solamente significa reconocer dos cosas:

En primer lugar, que, dada la sociabilidad natural del hombre, las interacciones sociales intervienen poderosamente en la configuración de la disposición y de la personalidad moral (Múgica, 2005a: 11). El modo en que lo hacen puede ser 
explicado de distintas maneras, pero en todo caso supone una apropiación de tal fin por parte del sujeto. En este punto cobra importancia el concepto de «representación», que si bien adquiere más peso en el último Durkheim —el cual habla profusamente de representaciones colectivas (Pickering, 2000: 2; Mestrovic, 1991: 91) - se encontraba ya también en la DTS, donde concibe la representación principalmente en términos de «fuerza» impulsora de un proceso fisiológico y psicológico, el cual, a su vez, suscita una variedad de contenidos psíquicos, principalmente emocionales (Durkheim, 1967: 86-7).

Por esta vía Durkheim cree poder explicar la génesis de ciertos movimientos sociales, así como la institución del castigo (Durkheim, 1967: 88-9): "la naturaleza de los sentimientos colectivos manifiesta la pena y, en consecuencia, el crimen» (Durkheim, 1967: 92). En ello va indicado, en segundo lugar, que la referencia al orden social interviene en la definición de ciertas conductas morales. Ya hemos visto que, para Durkheim, las conductas sancionadas por el derecho penal son reflejo de una solidaridad social basada en las semejanzas —solidaridad mecánica (Durkheim, 1967: 93-4)—, mientras que el derecho restitutivo o correctivo se hace cargo de una solidaridad de tipo orgánico.

Subrayar esto tiene importancia porque, al lado de los elementos que definen la pertenencia de un individuo a una sociedad, en todo individuo encontramos, además, elementos que le diferencian del resto, elementos que configuran su personalidad individual (Durkheim, 1967: 93-4). Esto último también encuentra su reflejo en otras reglas morales, así como en aquellas reglas del derecho "que se ubican en regiones muy excéntricas» a la conciencia común —representadas en el derecho penal- Con todo, también estas dimensiones «excéntricas» interesan a la sociedad; de lo contrario, «el derecho restitutivo no tendría nada en común con la solidaridad social, pues las relaciones que regula, enlazarían a los individuos unos a otros sin ligarlos a la sociedad. Serían simples acontecimientos de la vida privada, como lo son, por ejemplo, las relaciones de amistad. Pero la sociedad dista mucho de estar ausente de esta esfera de la vida jurídica (...) Todo contrato supone... que detrás de las partes que se comprometen, está la sociedad lista para intervenir y hacer respetar los compromisos contraídos... ella sólo otorga fuerza obligatoria a los contratos que tienen por sí mismos un valor social» (Durkheim, 1967: 81-2).

Es decir: la sociedad, como instancia sancionadora del orden moral, no está ausente siquiera de esas relaciones que, en apariencia, implican únicamente a agentes particulares. Ella es la que, en último término, proporciona respaldo coercitivo a los pactos, las promesas y contratos, que constituyen la materia ordinaria de la justicia correctiva, en la que se ven implicados agentes particulares (individuales o colectivos).

Si la insistencia en el elemento coercitivo vincula a Durkheim con la filosofía moral moderna 5 , la idea que trata de expresar no es nueva. En términos aristotélicos podríamos decir que si bien las relaciones de justicia entre las partes - ya sea justicia correctiva o distributiva - tienen por objeto directo el bien de otro

\footnotetext{
5 En Hume la justicia se define antes de la aparición del gobierno, pero éste aparece para sancionar esas relaciones. En Kant el Estado viene también a asegurar las relaciones de justicia preexistentes.
} 
particular, de hecho tales relaciones se enmarcan en el contexto de la justicia política, que supone la institución de la ley por referencia al bien común de la sociedad.

Ciertamente, a la hora de caracterizar con precisión la naturaleza moral del vínculo social importa distinguir las obligaciones que tienen por objeto a la sociedad en su conjunto de aquellas otras que tienen por objeto a particulares, por mucho que también estas últimas afecten en último término al conjunto. De lo contrario, correríamos el riesgo de confundir el ámbito de la acción individual con el de la acción social propiamente dicha. En este punto, puede ser relevante la reflexión que hace Tomás de Aquino al comienzo de su Comentario a la Ética a Nicómaco: el todo social no es un todo continuo, sino un todo de orden, de tal manera que, mientras que por un lado, las partes que componen dicha sociedad pueden realizar operaciones que no se identifican sin más con la operación del todo (no todo lo que hace el miembro de la familia es eo ipso acción de la familia), por otro hay acciones de los miembros de la sociedad que sí valen como acciones de la sociedad en su conjunto: por ejemplo, cuando un soldado toma parte en la acción defensiva del ejército (Tomás de Aquino, 1964, 4, n.5).

Ahora bien, en la medida en que los individuos humanos son naturalmente sociales, las mismas acciones que realizan a título individual no son ajenas al orden social —familiar, político- en el que se desenvuelven: a fin de cuentas, al actuar, los individuos buscan su bien, y éste depende en parte del orden social del que se benefician y al que deben contribuir, no sólo de manera negativa — no causando daño- sino también de manera positiva, realizando ciertas acciones. Precisamente aquí se advierte la relevancia de incluir la perspectiva de los deberes en la consideración del orden social.

En este punto tal vez interese resaltar que pensar el orden social desde esta perspectiva no va en detrimento de la aproximación desde la virtud. La afirmación aristotélica según la cual la buena acción — la acción según virtud- es en sí misma un fin (Aristóteles, 1985: 90,92; EN VI, 2, 1139b; 1140b) -y no simplemente un medio para otra cosa, llamada felicidad-, puede considerarse equivalente a la afirmación kantiana según la cual hay fines que es un deber tener (Kant, 1989: 232ss; MS, 6: $382 \mathrm{ss)} \mathrm{—los} \mathrm{cuales} \mathrm{constituyen,} \mathrm{precisamente,} \mathrm{deberes} \mathrm{de} \mathrm{virtud.}$

Al mismo tiempo, es cierto que la perspectiva de las obligaciones mutuas no agota el ámbito de la moral, pues, aunque permite dar cuenta de su estructura no termina de explicar su dinamismo, el cual sólo se explica porque de hecho los hombres buscan su bien, o, si se quiere, buscan su felicidad. Durkheim no desconoce este aspecto dinámico de la vida moral; por ello al lado de la obligatoriedad derivada de la estructura social, y que está en la base de la moral de la cooperación, del solidarismo moderno, advirtió la necesidad de considerar lo deseable como motor de la conducta individual. Mestrovic hace notar cómo en un ensayo titulado «La determinación del hecho moral» (1906), Durkheim se destaca de Kant precisamente en este punto:

«La noción de deber no agota el concepto de lo moral. Es imposible para nosotros realizar un acto simplemente porque se nos ordena hacerlo y sin consideración de su contenido. Para ser agentes de un acto, éste debe interesar a nuestra sensibilidad en alguna medida y aparecérsenos, de algún modo, como algo deseable. La obligación o el deber expresa únicamente un aspecto abstracto de la moral. Un cierto grado de deseabilidad es la otra característica, no menos importante que la primera» (cit. Mestrovic, 1991: 122). 
Aunque aquí no vamos a continuar el análisis, es importante subrayar que ambos elementos —obligatoriedad y «deseabilidad», o, como señala Hall, motivación moral (Hall, 1993: 19) — son necesarios para reconstruir la unidad de la vida ética, empeño al que, desde joven, dirigió Durkheim sus esfuerzos (Joas, 1997; Múgica, 2005b: 27). Por eso el análisis de la estructura e instituciones sociales, ensayado por Durkheim en la División del Trabajo Social, tenía que completarse con un análisis de las condiciones psicológicas de la vida social y la génesis de nuevas instituciones: un movimiento hacia la interioridad exigido ya por los análisis que llevó a cabo en el Suicidio, que le llevaron a enfatizar progresivamente el concepto de «representación", hasta conducirle, en Las formas elementales de la vida religiosa, a explorar expresamente la cuestión de la conducta movida por ideales. Según Joas, en este punto el propósito de Durkheim no era otro que proporcionar una teoría empíricamente fundada de la experiencia y acción religiosa, con el fin de preservar esos modos de experiencia y acción en condiciones no religiosas (Joas, 1997: 241).

\section{Naturalismo ético y sociología}

En todo caso, la perspectiva de las obligaciones mutuas no solo es relevante para la consideración del orden social desde el punto de vista objetivo - los deberes institucionalizan exigencias básicas y funcionales derivadas de la vida y la estructura social - sino también porque tales obligaciones definen la materia sobre la que versa la acción moral del sujeto. Ambos aspectos quedan suficientemente recogidos en la teoría social de Durkheim. En lo que toca a la forma de la acción moral, sin embargo, la elaboración de Durkheim es más bien escasa. Aunque su recurso al concepto de «representación» —que en una obra tardía como la Educación Moral (1925) aparece incluso como condición de las normas sociales (Paoletti, 2012: 170) — permite dar algunos pasos en la dirección de una mayor definición de la forma de la acción moral, se echa en falta una explicación del modo en que tiene lugar la apropiación subjetiva de la norma, de tal manera que el agente no solo actúe bien sino que lo haga por el motivo debido.

¿Cuál sería, por lo demás, el motivo debido? Nos inclinamos a suponer que la acción moral, para Durkheim, sería aquella en la que el sujeto es máximamente receptivo al sentimiento moral y actúa desde él. Aquí podría verse la influencia de Schopenhauer. En el caso de Durkheim, la fuente de este sentimiento moral, no parece ser otra que la sociedad, aunque este juicio podría deberse en parte a las limitaciones que le impone su propio método, con el cual, según sus propias palabras, no pretende excluir una aproximación metafísica a la moral:

«Esta ciencia no está en oposición con ninguna especie de filosofía, pues se ubica en un terreno muy distinto. Es posible que la moral tenga algún fin trascendente que la experiencia no puede alcanzar; este asunto le corresponde al metafísico. Pero lo que es cierto, ante todo, es que se desarrolla en la historia y bajo el imperio de causas históricas, que tiene una función en nuestra vida temporal. $\mathrm{Si}$ es tal o cual en determinado momento, se debe a que las condiciones en las cuales viven entonces los hombres no permiten que sea de otra manera, y la prueba de esto es que cambia cuando cambian las condiciones, y sólo en este caso». (Durkheim, 1967: 33) 
Ciertamente, una vez que adoptamos las restricciones impuestas por el método empírico — que nos obliga a definir lo moral en atención a los rasgos de solidaridad y coerción-, entonces la sociedad aparece ya como la única fuente de la normatividad moral, no sólo en lo que se refiere a la forma de la acción, sino también en cuanto a su contenido: la norma moral nace en la sociedad sin otro fin que asegurar la solidaridad, y, en este sentido, la supervivencia de la misma sociedad. En ello puede reconocerse un sesgo estoico, por lo demás muy presente en toda la filosofía moderna, al menos desde Grocio: no es el bien como tal, sino la conservación de la sociedad, lo que da razón última de las normas morales. O, si se prefiere, el bien queda reducido a la conservación de la sociedad. En la medida en que el hombre, y todas sus dimensiones, se contemplan desde la óptica de la sociabilidad, o en función de ella, tal conclusión se impone por sí sola. Bien mirado, sin embargo, tal conclusión no deja de constituir un caso más de la falacia naturalista que el filósofo británico G. E. Moore reconoció en algunas teorías éticas —entre ellas la ética evolutiva de Spencer-: una falacia consistente en sustituir el predicado «bueno» - de suyo indefinido y simple-por un contenido concreto, en este caso la supervivencia del propio sujeto: "conatus sese conservandum esse essentia rerum», decía Espinosa.

Tal conclusión solo podría evitarse en la medida en que se restaurase el pensamiento teleológico negado por Espinosa, en la medida en que se volviera a distinguir fin (como sentido) y función, sin reducir aquél a ésta. Después de todo, ¿por qué habría de consistir el bien en conservar la propia esencia? Si la única razón para preservar la propia vida - tanto individual como social- es que encontramos en nosotros una fuerte inclinación en este sentido, entonces el debilitamiento de esta inclinación acaba con todas nuestras razones. Pero entonces, la ética se confundiría realmente con la física. Solo cuando el objeto inmediato de la inclinación se integra en un horizonte pleno de sentido trascendemos el ámbito de las causas y entramos en el de las razones para la acción; sólo si la esencia humana es portadora de algo absoluto, si apunta a algo absoluto, que trasciende toda consideración meramente funcional, tiene sentido empeñarse en su conservación.

De todos modos, el naturalismo de la propuesta durkheimiana — para Hall el principal defecto de la ética de Durkheim (Hall, 1993: 23) — es de una índole peculiar, porque no permite la reducción simple del bien moral a un único principio. Se trata, más bien, de un naturalismo complejo, que recuerda a Hegel por su intento de identificar la ética propia de una sociedad a partir de los valores incorporados en las instituciones, en la cultura. Tal vez por ello reviste especial interés el hecho de que, posteriormente, Durkheim se haga eco de Kant al afirmar el valor incondicional del individuo: todo individuo, portador de la humanidad, portador de la razón, es merecedor de incondicional respeto.

En este «individualismo moral», que afirma la sacralidad de la persona, Durkheim advierte el modo específico con el que la sociedad moderna, crecientemente secularizada, se dota de la legitimidad moral que ya no puede esperar de la religión socialmente compartida (Durkheim, 2002: 41-2). Este, como es sabido, era el tema de su Educación Moral, con la que perseguía defender una moral secular para su tiempo. Tal y como lo ha expresado Múgica, «el individualismo moral es la genuina expresión religiosa moderna»(2005c: 20-21). Robert Bellah ha apuntado las tensiones inherentes a este proyecto de una moral racional puramente secular 
(Bellah, 1973). Con todo, el intento de Durkheim es significativo de que, de un modo u otro, la moral no puede pasarse sin lo sagrado, de lo que se sustrae a, y va más allá de, cualquier consideración funcional.

\section{BibliografíA}

ARistóteles (1985), Ética a Nicómaco, ed.bilingüe por Maria Araujo y Julián Marías, Madrid,Centro de Estudios Constitucionales.

Bellah, R, (1973), «Introduction» to Emile Durkheim on Morality and Society, Chicago and London, The University of Chicago Press.

BerRy, C. (1997), Social Theory of the Scottish Enlightenment, Edinburgh, Edinburgh University Press.

Cooper, A. (1977), Reason and human good in Aristotle, Cambridge, Harvard University Press.

Durkheim, E. (2002), La educación moral, Madrid, ed. Morata.

Durkheim, E. (1967), De la división del trabajo social, Buenos Aires, ed. Schapire.

Durkheim, E. (1975), «La science positive de la morale en Allemagne», Revue Philosophique, 24/1887: 54; en Textes 1. Éléments d'une théorie sociale, París, ed. de Minuit.

Durkheim, E. (1992), L'education morale, París, PUF, $1^{\text {a }}$ ed. 'Quadrige».

GonzÁLEZ, A. M. (2004a), "Cultura y felicidad en Kant», Teorema, Volumen XXIII/1-3: 215232.

González, A. M. (2004b), «La doble aproximación de Kant a la cultura», Anuario Filosófico, $37 / 3, n^{\circ} 80: 679-711$

Gonź́Lez, A. M. (2008), «La justicia como virtud artificial en Hume: elementos para una teoría psico-social de la acción», Pensamiento, 69, 239: 97-127.

GonzÁlez, A. M. (2009), "Kant's contribution to social theory», Kant Studien, 100, 1: 77-105.

Gonź́LEz, A. M. (2010), «Kant and a culture of freedom», Archiv für rechts-und sozialphilosophie, vol. $96, \mathrm{n}^{\circ}$ 3: 291-308.

González, A. M. (2011a), Culture as mediation. Kant on nature, culture, and morality, Hildesheim, Olms.

González, A. M. (2011b), «Norma moral y cambio social. La aproximación de los filósofos», Acta Philosophica, vol. 20, 2: 243-271.

González, A. M. (2013), Sociedad civil y normatividad. La teoría social de David Hume, Madrid, Dykinson, 2013.

González, A. M. (2014), «Kant on history», Estudos Kantianos, vol. 2, nº 2: 265-290.

González, A. M. (2016a), «The Recovery of Action in Social Theory: Acting out of Sentiment, Acting out of Character, Acting out of Interest, Acting out of Will», in Alznauer, M. \& Torralba, J.M. (eds), Theories of Action and Morality. Perspectives from Philosophy and Social Theory, Hildesheim, Zürich, New York, Georg Olms Verlag, pp. 79-111.

González, A. M. (2016b), La articulación ética de la vida social, Granada, Comares.

Hall, R. T. (1993), "Introduction», pp. 11-53 in Ethics and the Sociology of Morals. Emile Durkheim, Amherst, New York, Prometheus Books.

JoAs, H. (1997), «Durkheim's intellectual development», pp. 229-245, in Emile Durkheim. Socio-logist and moralist, edited by Stephen P. Turner, Routledge, London, New York.

KANT, I., 1985, La Metafísica de las Costumbres, traducción de Adela Cortina y Jesús Conill, Madrid, Tecnos.

KANT, I. (1994 a), Idea para una historia universal con propósito cosmopolita, Tr. C. Roldán y R. Rodríguez Aramayo, Madrid, Tecnos, $2^{\mathrm{a}}$ ed.

KanT, I. (1994 b), «Replanteamiento sobre la cuestión de si el género humano se halla en continuo progreso hacia lo mejor», pp. 79-100, en Idea para una historia universal con propósito cosmopolita, Tr. C. Roldán y R. Rodríguez Aramayo, Madrid, Tecnos, $2^{\mathrm{a}}$ ed. 
LukEs, S. (1984), Émile Durkheim. Su vida y su obra. Estudio histórico-crítico, Madrid, Siglo XXI, (1 ${ }^{\mathrm{a}}$ ed. or., 1973)

Mestrovic, S. G. (1991), The coming fin de siecle. An application of Durkheim's Sociology to modernity and postmodernism, London, New York, Routledge.

Mestrovic, S. G. (1992), Durkheim and postmodern culture, New York, Aldine de Gruyter.

MılL, J. S. (2002), «El utilitarismo». Un sistema de la lógica, libro VI, cap. XII, tr. E. Guisán, Madrid, Alianza.

Múgica, F. (2004a), Émile Durkheim. Civilización y división del trabajo (I). Ciencia social y reforma moral, Pamplona

Múgica, F. (2004b), Émile Durkheim. Civilización y división del trabajo (II). La naturaleza moral del vínculo social, Pamplona.

Múgica, F. (2005a), Émile Durkheim, Civilización y división del trabajo (III). Cambio social e individualismo moral, Pamplona.

Múgica, F. (2005b), Émile Durkheim. La constitución moral de la sociedad (I). El marco normativo de la acción social, Pamplona.

Múgica, F. (2005c), Émile Durkheim. La constitución moral de la sociedad (III). Los elementos de la moralidad y la configuración social de la vida ética. Pamplona.

Pickering, W. S. F. (ed.) (2000), Durkheim and Representations, London, Routledge.

PInTo, L., 2009, La théorie souveraine. Les philosophes français et la sociologie au XX siècle, Passages, París, Les Éditions Du Cerf.

Paoletti, G. (2012), Durkheim et la philosophie. Représentation, réalité et lien social, Paris, Classiques Garnier.

SPEncer. H. (1966), The Principles of Sociology, III, The Works of Herbert Spencer, Osnabrück, Otto Zeller, 1966, vol. VIII

Tomás de Aouino (1964), In decem Libros Ethicorum Aristotelis ad Nicomachum Expositio, a cura di Raymundi M. Spiazzi, Taurini, Marietti, $3^{\text {a }}$ ed.

Tomás de Aouino (199)7, Suma de Teología IV, Parte II-II(b), Madrid, Bac, $2^{\text {a }}$ ed.

Turner, B. S. (1996), «Preface», en Durkheim, E., Professional Ethics and Civil Morals, London, New York, Routledge.

Universidad de Navarra

Ana María GonZÁlez

agonzalez@unav.es

[Artículo aprobado para publicación en diciembre de 2013] 
